\title{
Autoimmune Hepatitis Overlapping with Primary Sclerosing Cholangitis
}

\author{
Junko Takiguchi, Hiromasa Ohira, Tsuyoshi RaI, Shoichiro Shishido, Jun Tojo, Yukio Sato, \\ Reiji Kasukawa, Hiroshi Watanabe*, Yuji Funabashi* and Hiromi Kumakawa*
}

\begin{abstract}
A case of autoimmune hepatitis (AIH) complicated by primary sclerosing cholangitis (PSC) in a 36-year-old woman is reported. AIH overlapping with PSC has been rarely reported, and to the best of our knowledge, there have been no reports in Japan. Based on the criteria for diagnosis of AIH and on typical endoscopic retrograde cholangiograph (ERC) findings, the patient was diagnosed as having AIH overlapping with PSC. Her transaminase levels normalized within 1 month after administration of prednisone, azathioprine and ursodeoxycholic acid, but her cholestatic enzyme level remained elevated. The effect of treatment on the cholestatic features is thought to be an important factor for predicting the prognosis of AIH overlapping with PSC.

(Internal Medicine 41: 696-700, 2002)
\end{abstract}

Key words: AIH, PSC, endoscopic retrograde cholangiograph, prednisone, azathioprine, ursodeoxycholic acid

\section{Introduction}

Autoimmune hepatitis (AIH) is a chronic necro-inflammatory disorder of unknown etiology. The diagnosis is based on criteria that include clinical symptoms, hypergammaglobulinemia with an absence of cirrhosis, and circulating autoantibodies (1). Primary sclerosing cholangitis (PSC) is a cholestatic disease of unknown etiology characterized by diffuse inflammation and fibrosis of the intrahepatic and extrahepatic bile ducts (2). A syndrome in which $\mathrm{AIH}$ and primary biliary cirrhosis (PBC) overlap is often seen (3), but there have only been a few reports of an overlap between AIH and PSC (4-13). We describe here a case of AIH associated with PSC in a female patient who fulfilled criteria for definite AIH (14) and had characteristic cholangiographic features in the biliary tracts.

\section{Case Report}

In June 1993, a 36-year-old woman was admitted to another hospital because of low-grade fever. She had no history of blood transfusion or drug allergy, and she did not drink alcohol. Laboratory data on admission are shown in Table 1. Moderate increases in aspartate aminotransferase (AST: $381 \mathrm{IU} / l$ ), alanine aminotransferase (ALT: $264 \mathrm{IU} / l$ ) and alkaline phosphatase (ALP: 28.8 KAU) were noted. Serum IgG was elevated to 3,210 $\mathrm{mg} / \mathrm{dl}, 1.7$-times more than the upper limit of the normal range. Antinuclear antibody (ANA: 1: 1,280) was positive. Histology of a liver biopsy sample revealed findings of chronic active hepatitis without cholangitis, as shown in Fig. 1. On the basis of these findings, she was diagnosed as having autoimmune hepatitis and was treated with prednisone at a dose of $30 \mathrm{mg} /$ day and ursodeoxycholic acid (UDCA) at a dose of $600 \mathrm{mg}$ / day. Since the ALT level decreased gradually after the start of treatment, the administration of prednisone was stopped on her own will in December 1993. However, marked increases in ALT and ALP were noted in February 1994. She was then treated with prednisone $(50 \mathrm{mg} /$ day $)$ and azathioprine $(100 \mathrm{mg}$ / day), and the ALT level decrased. Since a moderate increase in ALP continued, she was referred to our department, and she was admitted in April 1994. Her status on admission was as follows: height, $152.0 \mathrm{~cm}$; body weight, $48.2 \mathrm{~kg}$; blood pressure, $120 / 80 \mathrm{mmHg}$; and pulse, regular at 84 beats/min. No goiter or enlargement of the cervical lymph nodes was noted. No remarkable abnormalities in the heart or lungs were noted. The liver edge was palpable three fingerbreadths under the right costal edge without tenderness. Laboratory data on admission are shown in Table 1. Moderate increases in ALP (893 IU/l) and $\gamma$-glutamyl transpeptidase ( $\gamma$-GTP: $373 \mathrm{IU} / l)$ were noted. The serum immunoglobulin level was not elevated. Anti-mitochondrial antibody (AMA) and anti-smooth muscle antibody (aSMA) were not detected by indirect immunofluorescence. She was positive for ANA $(1: 1,280)$ and perinuclear anti-neutrophil cytoplasmic antibody (pANCA). The results of tests for hepatitis B virus surface (HBs)-antigen, hepatitis B virus

From the Second Department of Internal Medicine, Fukushima Medical University School of Medicine, Fukushima and *the Department of Medicine, Soma General Hospital, Fukushima

Received for publication December 21, 2001; Accepted for publication April 30, 2002

Reprint requests should be addressed to Dr. Junko Takiguchi, the Second Department of Internal Medicine, Fukushima Medical University School of Medicine, 1-Hikarigaoka, Fukushima 960-1295 
AIH Overlapping with PSC

Table 1. Laboratory Data in Our Patient

\begin{tabular}{lcc}
\hline & Jun-93 & Apr-94 \\
\hline WBC & $5,300 / \mu l$ & $4,700 / \mu l$ \\
RBC & $351 \times 10^{4} / \mu l$ & $361 \times 10^{4} / \mu l$ \\
PLT & $\mathrm{ND}$ & $24.3 \times 10^{4} / \mu \mathrm{l}$ \\
AST & $381 \mathrm{IU} / l$ & $99 \mathrm{IU} / l$ \\
ALT & $264 \mathrm{IU} / l$ & $65 \mathrm{IU} / l$ \\
LDH & $460 \mathrm{IU} / l$ & $280 \mathrm{IU} / l$ \\
ALP & $28.8 \mathrm{KU}(\mathrm{normal} \mathrm{range} 2.2-10)$ & $893 \mathrm{IU} / l(\mathrm{normal} \mathrm{range} 125-335)$ \\
$\gamma$ GTP & $349 \mathrm{IU} / \mathrm{l}$ & $373 \mathrm{IU} / l$ \\
TB & $1.41 \mathrm{mg} / \mathrm{dl}$ & $0.7 \mathrm{mg} / \mathrm{dl}$ \\
DB & $0.86 \mathrm{mg} / \mathrm{dl}$ & $0.3 \mathrm{mg} / \mathrm{dl}$ \\
TP & $8.6 \mathrm{mg} / \mathrm{dl}$ & $7.6 \mathrm{mg} / \mathrm{dl}$ \\
Alb & $3.8 \mathrm{mg} / \mathrm{dl}$ & $4 \mathrm{mg} / \mathrm{dl}$ \\
TTT & $27.7 \mathrm{KU}$ & $27.3 \mathrm{KU}$ \\
ZTT & $31.9 \mathrm{KU}$ & $14.3 \mathrm{KU}$ \\
IgG & $3,210 \mathrm{mg} / \mathrm{dl}$ & $1,850 \mathrm{mg} / \mathrm{dl}$ \\
IgA & $213 \mathrm{mg} / \mathrm{dl}$ & $230 \mathrm{mg} / \mathrm{dl}$ \\
IgM & $404 \mathrm{mg} / \mathrm{dl}$ & $332 \mathrm{mg} / \mathrm{dl}$ \\
ANA & $\times 1,280(\mathrm{ho})$ & $\times 1,280(\mathrm{ho}, \mathrm{sp})$ \\
AMA & $(-)(\mathrm{M} 2)$ & $(-)$ \\
p-ANCA & $\mathrm{ND}$ & $\times 1,280$ \\
CH50 & $69.2 \mathrm{U} / \mathrm{ml}$ & $56.2 \mathrm{U} / \mathrm{ml}$ \\
\hline & &
\end{tabular}

ND: not done.

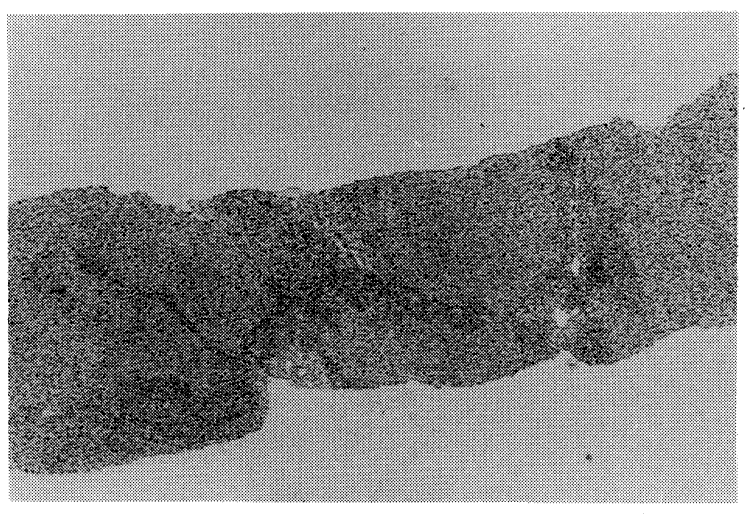

A

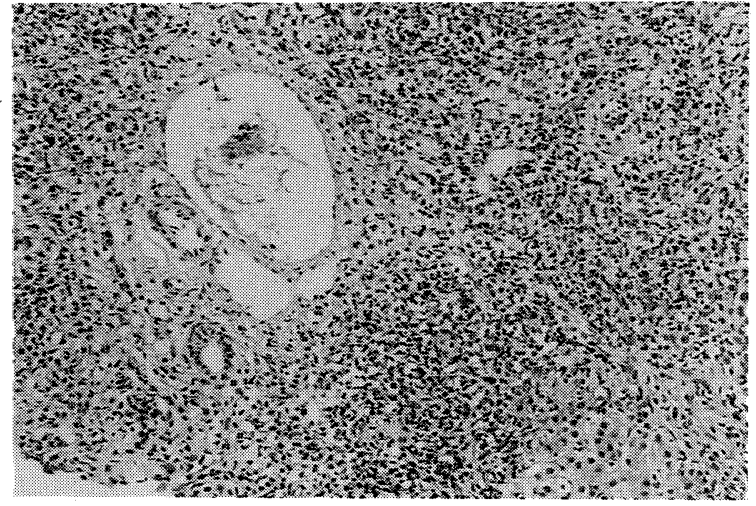

B

Figure 1. A, B. Histological findings in a liver biopsy specimen taken on June 5, 1993. A portal tract is infiltrated with an increased number of lymphocytes and shows piecemeal necrosis. A widened portal area with extensive fibrosis and partly bridging fibrosis is also noted. Focal necrosis is seen in the parenchyma. However, there are no findings such as periductal fibrosis, bile duct damage or loss of bile ducts $($ HE stain, A: $\times 10, B: \times 200)$.

core ( $\mathrm{HBc}$ )-antibody and hepatitis $\mathrm{C}$ virus $(\mathrm{HCV})$-antibody were all negative. Her human leukocyte antigen (HLA) typing was positive for A24, A31, B35, B61, Cw4 and DR4.

Endoscopic retrograde cholangiography (ERC) revealed shortening and irregularities of intra- and extrahepatic bile ducts compatible with PSC (Fig. 2). There was no evidence of inflammatory bowel disease on colonoscopic examination. A liver biopsy performed again in May 1994 showed significant amelioration of hepatitis with mild residual inflammatory infiltrate in the portal tracts but no evidence of cholangitic changes (Fig. 3). Based on the criteria for diagnosis of AIH and on the ERC findings, she was diagnosed as having AIH overlapping with PSC. One year later, her ALT and IgG levels had normalized but ALP and $\gamma$-GTP levels remain elevated. She has since been 


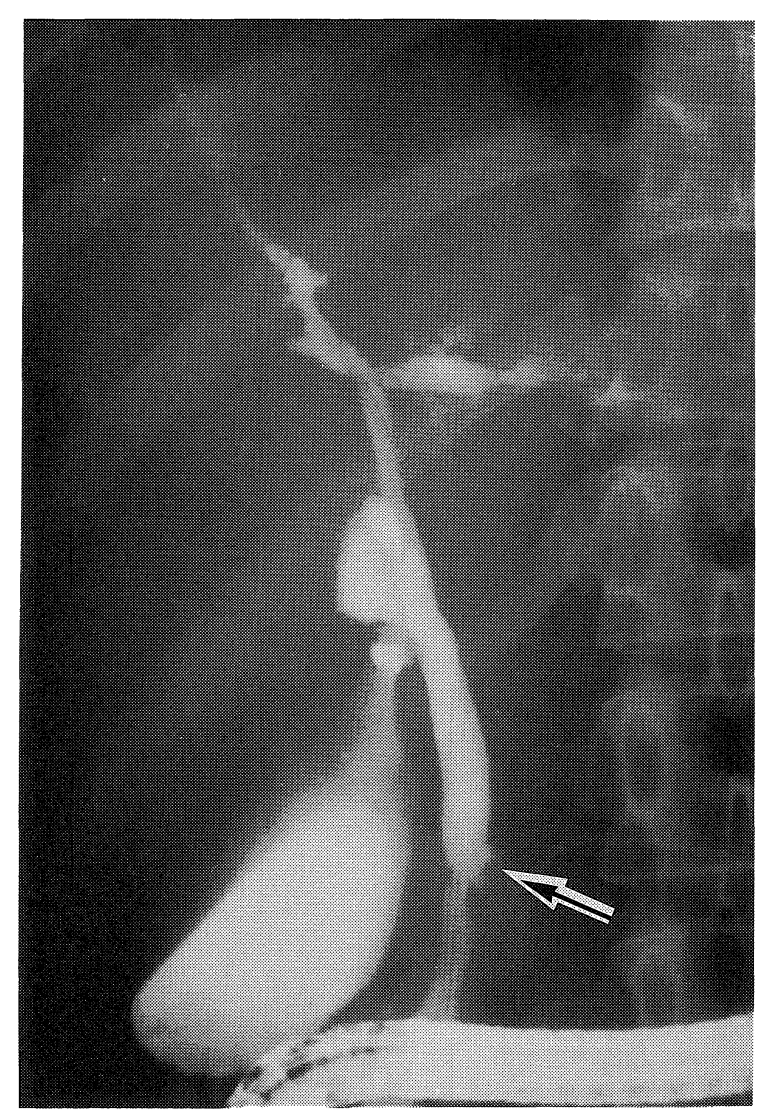

Figure 2. Endoscopic retrograde cholangiography reveals a mixture of narrowing and dilatation of both intra- and extrahepatic bile ducts. A diverticula finding (arrow) is observed at the common bile duct. treated with prednisone $(7.5 \mathrm{mg} / \mathrm{day})$ and azathioprine (100 $\mathrm{mg} /$ day).

\section{Discussion}

The initial diagnosis of AIH seems quite sound in this case. According to the revised criteria for diagnosis proposed by the International Autoimmune Hepatitis Group (14), the AIH score was calculated to be 18 , defined as 'definite' AIH. Histologically, the liver biopsy specimen showed interface hepatitis that was compatible with AIH. On the other hand, the diagnosis of PSC in this case was made on the basis of typical cholangiographic findings in the setting of cholestatic liver enzyme elevations, although the liver biopsy specimen showed no cholangitic findings or periductal fibrosis. Currently, the diagnostic criteria for PSC are based on characteristic cholangiographic findings, and a liver biopsy is not generally required to establish a diagnosis of PSC (15). In fact, the frequency of the fibrous-obliterative cholangitis in liver biopsy specimens is only about $10-50 \%$ in patients with PSC (16-18). Therefore, she was diagnosed as having AIH overlapping with PSC. After the diagnosis, the AIH score was recalculated to be 23 , defined as 'definite' AIH.

AIH overlapping with PSC has rarely been reported; to the best of our knowledge, only 27 cases have been reported (see Table 2). There appear to have been no reports in Japan. In the 27 documented cases of AIH overlapping with PSC, PSC was diagnosed before the onset of AIH in 3 cases, at the same time as the onset of AIH in 6 cases, and after the onset of AIH in 18 cases. These overlapping cases have been observed more frequently in young men (15/27), although AIH occurs most frequently in women between the ages of 10 and 30 years or in late middle age (19). An association of inflammatory bowel diseases was reported in 19 of the 27 cases. Although ulcerative colitis is complicated by AIH without PSC, the presence

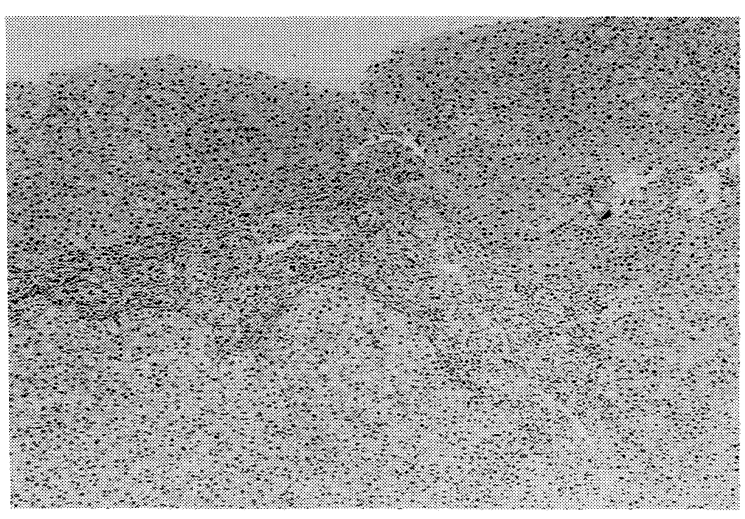

A

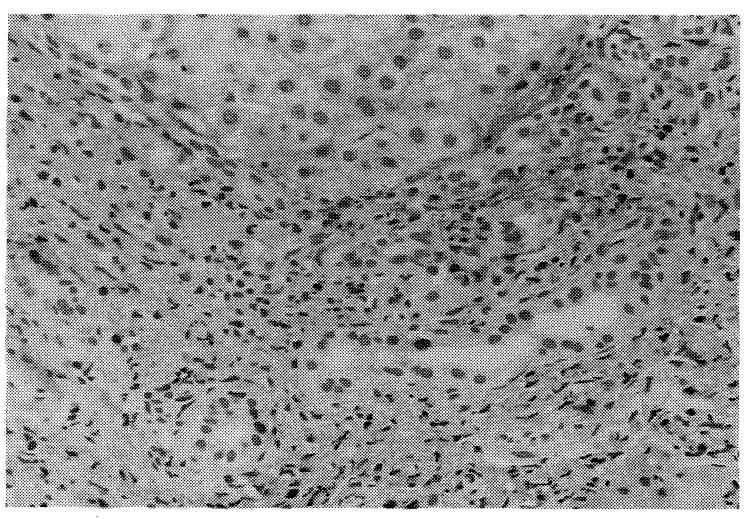

$\mathrm{B}$

Figure 3. A, B. Histological findings in a liver biopsy specimen taken on April 1, 1994. A portal tract is infiltrated with lymphocytes but to a lesser degree than that observed in the previous liver biopsy specimen (Fig. 1A). There are no findings such as periductal fibrosis, bile duct damage or loss of bile ducts (HE stain, A: $\times 40, B: \times 400)$. 
AIH Overlapping with PSC

Table 2. Clinical and Laboratory Characteristics of Published Cases with AIH/PSC Overlap Syndrome

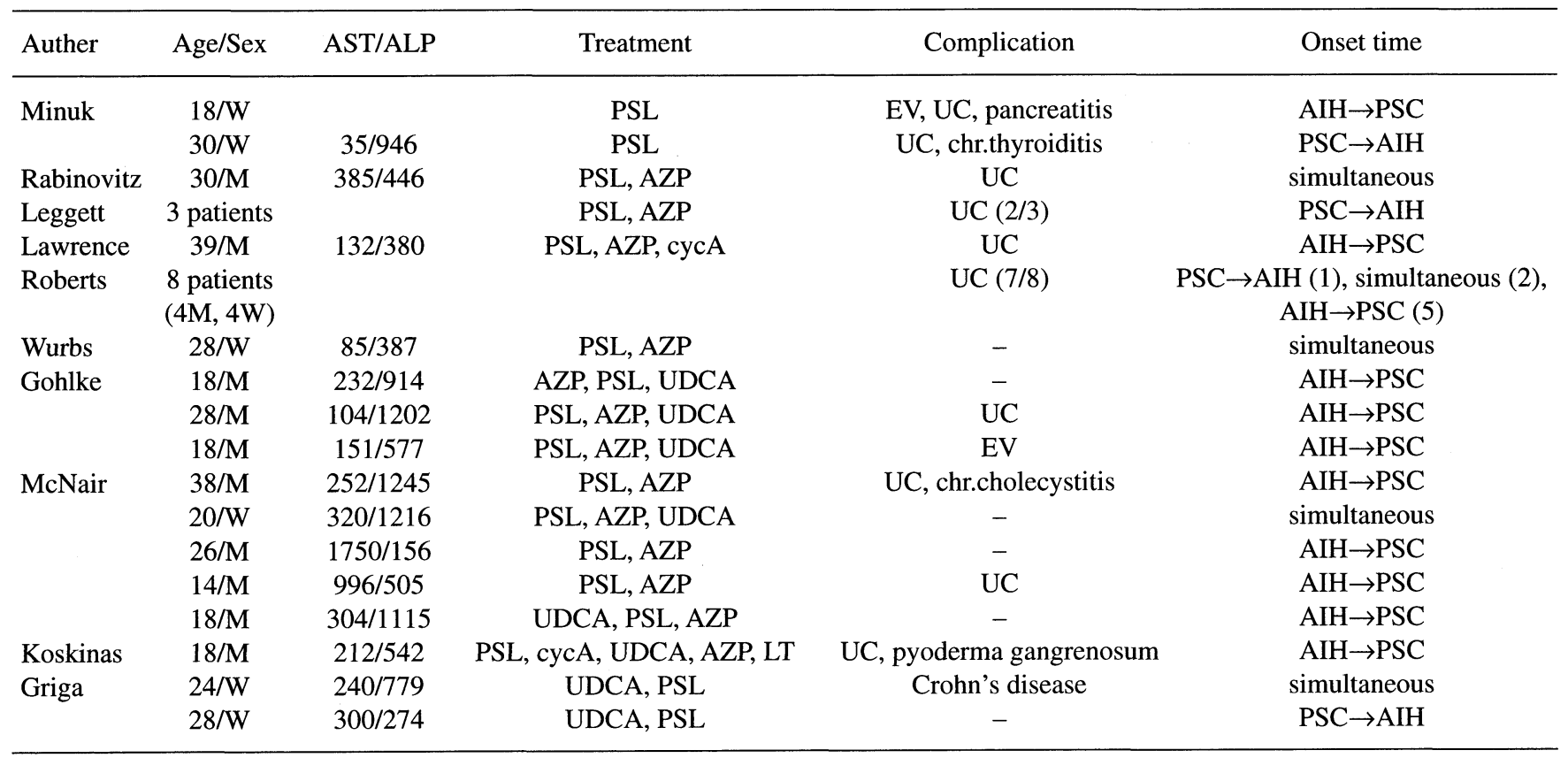

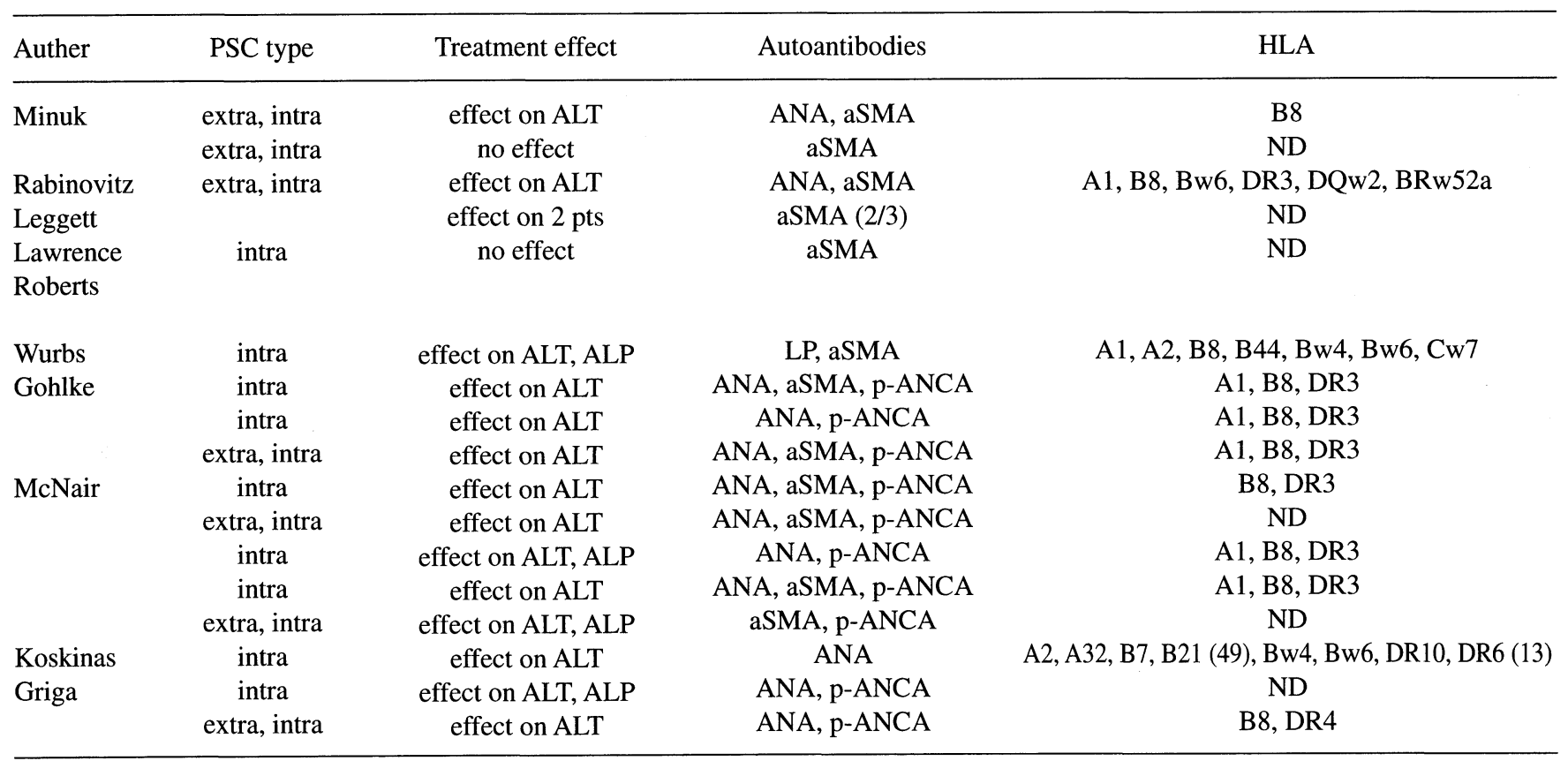

CsA: ciclosporin, LT: liver transplantation, EV: esophageal varices, UC: ulcerative colitis, LP: anti-liver-pomcreas antibody, ND: not done.

of PSC seems to raise the frequency of association of inflammatory bowel diseases. There was no evidence of inflammatory bowel disease in our case. One possible explanation for this is that the characteristics of PSC patients in Japan are different from those in other countries. The incidence of associated inflammatory bowel disease in Japanese patients with PSC is $21 \%$, which is lower than that reported in other countries (20).

The nature of the relationship between AIH and PSC is not known, although both diseases are believed to be caused by autoimmune reactions. As an abnormality of immunogenetic background, an association of HLA has been reported for both diseases. Previous studies have shown that both AIH and PSC are associated with HLA B8 and DR3 $(19,21)$, suggesting a common genetic basis. As shown in Table 2, B8-DR3 haplotype was observed in $77.8 \%$ of (7/9) AIH cases overlapping with PSC. Our case was positive not for B8-DR3 haplotype but for DR4, which is associated with a high degree of suscep- 


\section{TAKIGUCHI et al}

tibility to disease in Japanese AIH patients (22). However, the relationship between HLA-DR4 and AIH overlapping with PSC is uncertain, because there have been no reports of AIH overlapping with PSC in Japan. In addition, autoantibodies such as ANA, aSMA and pANCA and hypergammaglobulinemia are found in both diseases. However, these markers show no disease specificity and can not explain the cause of AIH overlapping with PSC.

Immunosuppressive drugs such as prednisone, azathioprine and cyclosporine have been used for treating cases of AIH overlapping with PSC. The response of immunosuppressive therapy observed for normalization of ALT level, whereas ALP level may be amenable to UDCA (10). In our case, the ALT level normalized within 1 month after administration of prednisone, azathioprine, and UDCA, but the cholestatic enzyme level remained elevated. Since a good response to immunosuppressive therapy is observed in patients with hepatitis-like features, it is conceivable that the effect of treatment on cholestatic features is an important factor for predicting the prognosis of overlap of AIH and PSC. Diagnosis of this overlap syndrome and close monitoring are essential for determining the optimal time for liver transplantation.

\section{References}

1) Johnson PJ, McFarlane IG. Meeting report of the International Autoimmune Hepatitis Group. Hepatology 18: 998-1005, 1993.

2) Wiesner RH, LaRusso NF. Clinicopathologic features of the syndrome of primary sclerosing cholangitis. Gastroenterology 79: 200-206, 1980.

3) Klöppel G, Seifert G, Lindner H, Dammermann R, Sack HJ, Berg PA. Histopathological features in mixed types of chronic aggressive hepatitis and primary biliary cirrhosis. Virchows Arch Pathol Anat 373: 143-160, 1977.

4) Minuk GY, Sutherland LR, Pappas SC, Kelley JK, Martin SE. Autoimmune chronic active hepatitis (lupoid hepatitis) and primary sclerosing cholangitis in two young adult females. Can J Gastroenterol 2: 22-27, 1988.

5) Rabinovitz M, Demetris AJ, Bou-Abboud CF, Van Thiel DH. Simultaneous occurrence of primary sclerosing cholangitis and autoimmune chronic active hepatitis in a patient with ulcerative colitis. Dig Dis Sci 37: 1606-1611, 1992.

6) Leggett BA, Hallam A, Powell LW. Autoimmune chronic active hepatitis evolving to primary sclerosing cholangitis: evidence for a common patho- genesis. Hepatology 16: 191A, 1992.

7) Lawrence SP, Sherman Ke Lawson JM, Goodman ZD. A 39-year-old man with chronic hepatitis. Semi Liver Dis 14: 97-105, 1994.

8) Roberts S, Poterucha JJ, Gore GJ, Czaja AJ. Overlap between type 1 autoimmune hepatitis and primary sclerosing cholangitis. Hepatology 22: 129A, 1995.

9) Wurbs D, Klein R, Terracciano LM, Berg PA, Bianchi L. A 28-year-old woman with a combined hepatic/cholestatic syndrome. Hepatology 22: 1598-1605, 1995 (clinical conference).

10) Gohlke F, Lohse AW, Dienes HP, et al. Evidence for an overlap syndrome of autoimmune hepatitis and primary sclerosing cholangitis. J Hepatol 24: 699-705, 1996.

11) McNair AN, Moloney M, Portmann BC, Williams R, McFarlane IG. Autoimmune hepatitis overlapping with primary sclerosing cholangitis in five cases. Am J Gastroenterol 93: 777-784, 1998.

12) Koskinas J, Raptis I, Manika Z, Hadziyannis S. Overlapping syndrome of autoimmune hepatitis and primary sclerosing cholangitis associated with pyoderma gangrenosum and ulcerative colitis. Eur J Gastroenterol Hepatol 11: 1421-1424, 1999.

13) Griga T, Tromm A, Muller KM, May B. Overlap syndrome between autoimmune hepatitis and primary sclerosing cholangitis in two cases. Eur J Gastroenterol Hepatol 12: 559-564, 2000.

14) Alvarez F, Berg PA, Bianch FB, et al. International Autoimmune Hepatitis Group report: review of criteria for diagnosis of autoimmune hepatitis. J Hepatol 31: 929-938, 1999.

15) Lazaridis KN, Wiesner RH, Porayko MK, Ludwig J, LaRusso NF. Primary sclerosing cholangitis, in: Schiff's Diseases of the Liver, Eighth Edition. Schiff ER, Sorrel MF, Maddrey WC, Eds. Lippincott-Raven Publishers, Philadelphia, 1999: 649-669.

16) Chapman RW, Arborgh BA, Rhodes JM, et al. Primary sclerosing cholangitis: a review of its clinical features, cholangiography, and hepatic histology. Gut 21: 870-877, 1980.

17) Barbatis C, Grases P, Shepherd HA, et al. Histological features of sclerosing cholangitis in patients with chronic ulcerative colitis. J Clin Pathol 38: 778-783, 1985.

18) Dickson ER, LaRusso NF, Wiesner RH. Primary sclerosing cholangitis. Hepatology 4 (suppl 1): 33S-35S, 1984.

19) Manns MP. Autoimmune hepatitis, in: Schiff's Diseases of the Liver, Eighth Edition. Schiff ER, Sorrel MF, Maddrey WC, Eds. LippincottRaven Publishers, Philadelphia, 1999: 919-935.

20) Takikawa H, Manabe T. Primary sclerosing cholangitis in Japan-analysis of 192 cases. J Gastroenterol 32: 134-137, 1997.

21) Chapman RW. Role of immune factors in the pathogenesis of primary sclerosing cholangitis. Semi Liver Dis 11: 1-4, 1991.

22) Seki T, Kiyosawa K, Inoko H, Ota M. Association of autoimmune hepatitis with HLA-Bw54 and DR4 in Japanese patients. Hepatology 12: 13001304, 1990. 